\title{
Stage A
}

National Cancer Institute

\section{Source}

National Cancer Institute. Stage A. NCI Thesaurus. Code C28056.

Cancer confined to the original anatomic site of growth. 